\title{
Nurse-led cognitive behavioural therapy for treatment of anxiety in COPD
}

\author{
Abebaw Mengistu Yohannes (1) \\ School of Behavioral and Applied Sciences, Dept of Physical Therapy, Azusa Pacific University, Azusa, \\ CA, USA.
}

Correspondence: Abebaw Mengistu Yohannes, 701 East Foothill Boulevard, School of Behavioral and Applied Sciences, Dept of Physical Therapy, Azusa Pacific University, Azusa, 91702 CA, USA. E-mail: ayohannesđapu.edu

$@$ ERSpublications

Nurse-led cognitive behavioural therapy is feasible and cost-effective for the treatment of anxiety in patients with chronic obstructive pulmonary disease. http://ow.ly/QZJT30mJdXU

Cite this article as: Yohannes AM. Nurse-led cognitive behavioural therapy for treatment of anxiety in COPD. ERJ Open Res 2018; 4: 00221-2018 [https://doi.org/10.1183/23120541.00221-2018].

Anxiety disorders are common and debilitating conditions that significantly impair the physical functioning and quality of life (QoL) of patients with chronic obstructive pulmonary disease (COPD). A recent systematic review identified the high prevalence of these disorders in patients with COPD, ranging 6-33\% for generalised anxiety, $0-44 \%$ for panic disorder, $10-27 \%$ for specific phobias, and $5-11 \%$ for social phobias [1]. Furthermore, under-recognised or untreated anxiety disorders in COPD patients may reduce the QoL of their caregivers and increase emergency care visits and hospital admissions [1-5]. In 2004, in the European Union, the estimated direct (e.g. hospital admissions and medications) and indirect (e.g. loss of productivity due to absence from work) cost of anxiety disorders exceeded $€ 41$ billion per annum [2].

The exact mechanism of how anxiety disorders develop in patients with COPD is not clearly understood, but most likely includes multiple physiological, behavioural and social factors (table 1). Anxiety is characterised by elevated fatigue, irritability, poor concentration and poor sleeping patterns, a feeling of dysphoria or somatic symptoms of tension in patients with COPD [5-8]. Anxiety symptoms such as hyperventilation, frustration, anger and panic can, in turn, heighten the sensation of dyspnoea symptoms $[4,6,7]$. Growing evidence suggests that nonpharmacological intervention with pulmonary rehabilitation (PR) improves exercise capacity, QoL, and anxiety and depressive symptoms in patients with COPD [9, 10]. However, after completion of PR the benefits gradually wane in the succeeding months, partly due to a lack of maintenance therapy as patients fail to adhere to their exercise programmes.

Recent studies demonstrate the value of psychological therapy, with promising results for cognitive behavioural therapy (CBT) in the treatment of depression and anxiety in patients with COPD [11, 12]. CBT is "a time-sensitive, structured, present-oriented psychotherapy directed toward solving current problems and teaching clients skills to modify dysfunctional thinking and behaviour" [13]. This talking therapy is patient-centred and individualised, with specific goals of improving health behaviours and adherence to treatment. In patients with COPD, group CBT reduces patient-reported symptoms, increases exercise therapy, improves QoL, augments adherence to medical treatment and diminishes exacerbations $[14,15]$. Despite these benefits, CBT remains underused in COPD patients, partly due to limited access for patients to CBT, and primary care providers not referring patients for CBT due to a paucity of knowledge, excessive waiting time and inadequate provision of psychological services [16, 17]. Furthermore, access to trained CBT therapists in the national health service is scarce. Studies that have been conducted to deliver one-to-one CBT sessions using nurses are relatively small in sample sizes and their efficacy in the treatment of anxiety was inconclusive $[12,18,19]$.

Copyright $\odot$ ERS 2018. This article is open access and distributed under the terms of the Creative Commons Attribution Non-Commercial Licence 4.0. 


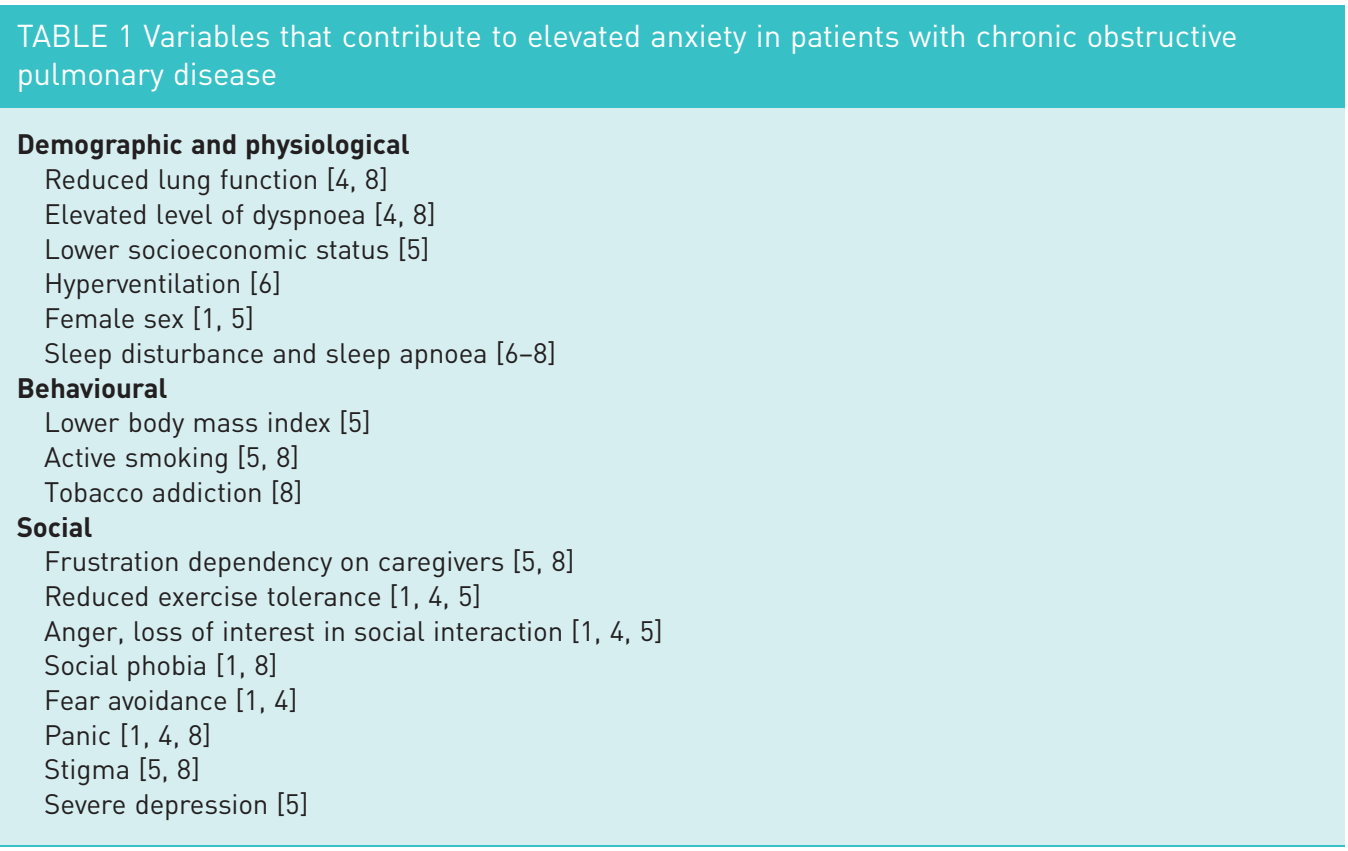

In ERJ Open Research, Heslop-Marshall et al. [20] report their findings from an adequately powered, randomised controlled trial of CBT compared to self-help leaflets to treat anxiety in patients with COPD. After 1 year of follow-up, CBT delivered by respiratory nurses (one-to-one therapy, for an average of four sessions) effectively reduced symptoms of anxiety, emergency care and hospital admissions in a cost-effective manner. Fewer respiratory admissions and emergency visits reduced the total mean cost for the CBT patients, more than offsetting the expense of providing CBT. Only the reduction in emergency visit costs, and not respiratory admission costs or total costs, achieved statistical significance due to variability. Nevertheless, a statistically significant improvement in quality-adjusted life-years favoured CBT, rendering intervention with CBT almost certainly cost-effective. HesLop-Marshall et al. [20] should be applauded for conducting this large study, and particularly for demonstrating that briefly trained nurses can supervise and implement effective, and almost certainly cost-effective, one-to-one CBT for patients with COPD.

What are the implications of the findings to healthcare professionals who are engaged in caring for patients with COPD and comorbid anxiety? First, CBT effectively ameliorates anxiety symptoms. Healthcare practitioners should consider CBT as a first-line treatment option for COPD patients with mild-to-moderate anxiety. Secondly, CBT appears to be a cost-effective treatment option compared to the usual care (leaflets). Thirdly, briefly trained nurses can deliver effective CBT.

Study strengths include its large sample size, adequately powering the randomised controlled trial design. Sessions were audio taped and scrutinised for fidelity, and a comprehensive cost analysis highlighted the value of CBT. But as acknowledged by the authors, some limitations of the study mean that findings should be interpreted with caution. First, the Hospital Anxiety Depression scale screens for anxiety and depressive symptoms. Therefore, significant anxiety disorders (e.g. panic and phobias, which are common in patients with COPD) were not fully examined using the gold standard of a structured psychiatric interview and the criteria of the Diagnostic and Statistical Manual of Mental Disorders. Secondly, it is encouraging to observe that four brief CBT sessions effectively ameliorated clinically meaningful anxiety symptoms, and sustained this benefit for 1 year. Similarly, a recent study demonstrated that a brief CBT intervention in a primary care setting for medically ill veterans with COPD and heart failure reduced comorbid anxiety and depressive symptoms over 1 year [11]. But whether a brief CBT intervention will remain efficacious over the lifetime of a chronic disease such as COPD remains unanswered. Thirdly, some uncertainty remains as to whether "off the shelf," self-help leaflets sufficiently provide and guide an active intervention. A recent systematic review and meta-analysis of 31 randomised, controlled trials demonstrated the efficacy of self-help in patients with anxiety compared to the waiting list [21]. Furthermore, another systematic review and meta-analysis showed that guided self-help was comparable to face-to-face psychotherapy intervention in reducing anxiety and depressive symptoms in patients with chronic diseases with 1-year follow-up [22]. However, caution need to be exercised when using self-help leaflets for severe and chronic anxiety disorders with a compromise of cognitive deficits and both sight and hearing impairments. 
How can we incorporate CBT in routine care? Clinicians should be aware of when to initiate CBT for anxiety disorders in patients with COPD, in relation to other treatment options such as pharmacotherapy (with antidepressants and/or anxiolytics) and PR. Notably in the study by HesLop-Marshall et al. [20], over one-third of COPD patients in both CBT and the leaflet control group received antidepressant drug therapy, and a small percentage also received anxiolytic medication, during the intervention period. In addition, over half of the patients had PR prior, during or following either the CBT or leaflet intervention. Thus, the study demonstrated real world clinical practice in the UK, and suggested that the benefit of CBT can be reaped at any point in the management of mild-to-moderate anxiety in patients with COPD.

Additional challenges exist for integrating CBT into routine clinical care. Inadequate resources are available to train adequate numbers of mental healthcare professionals to deliver CBT. HESLOP-MARSHALL et al. [20] show that primary care nurses can effectively deliver CBT with modest training. In addition, formal recognition of $\mathrm{CBT}$ in the reimbursement scheme for both general practitioners and respiratory specialists may facilitate the appropriate use of CBT for the treatment of anxiety disorders in patients with COPD. They have demonstrated the financial benefits gained by CBT intervention, with behavioural benefits lasting for a year, that also reduce emergency care and hospitalisations in a cost-effective manner.

Which patients are most likely respond to CBT? Patients with cardiopulmonary disease who exhibit greater physical disability and lower self-efficacy (unable to manage their symptoms and maintain functioning) are less likely to respond to anxiety and depressive symptoms after brief CBT [23]. Thus, future studies need to examine the efficacy of booster or maintenance CBT. Moreover, anxiety disorders confer a considerable degree of physical impairment, limited social interactions, dependency on caregivers, high healthcare use, and large societal economic burden due to absence from work [24]. Thus, CBT may be the first step towards reducing cost and improving QoL in patients with COPD.

In summary, Heslop-Marshall et al. [20] indicate that brief CBT is feasible and cost-effective for the treatment of anxiety in patients with COPD. Healthcare delivery systems should look for the means to provide this effective therapy to COPD patients.

Conflict of interest: None declared.

\section{References}

1 Willgoss TG, Yohannes AM. Anxiety disorders in patients with COPD: a systematic review. Respir Care 2013; 58: 858-866.

2 Andlin-Sobocki P, Wittchen HU. Cost of anxiety disorders in Europe. Eur J Neurol 2005; 12: Suppl. 1, 39-44.

3 Dahlen I, Janson C. Anxiety and depression are related to the outcome of emergency treatment in patients with chronic obstructive pulmonary disease. Chest 2002; 122: 1633-1637.

4 Livermore N, Sharpe L, McKenzie D. Catastrophic interventions and anxiety sensitivity as predictors of panic spectrum psychopathology in chronic obstructive pulmonary disease. J Psychosom Res 2012; 72: 388-392.

5 Yohannes AM, Baldwin RC, Connolly MJ. Depression and anxiety in elderly outpatients with chronic obstructive pulmonary disease: prevalence and validation of the BASDEC screening questionnaire. Int J Geriatr Psychiatry 2000; 15: 1090-1096.

6 Hannink J, Lahaije A, Bischoff E, et al. Dynamic hyperinflation after metronome-paced hyperventilation in COPD - a 2 year follow-up. Respir Med 2010; 104: 1700-1705.

7 Ferguson CJ, Stanley M, Souchek J, et al. The utility of somatic symptoms as indicators of depression and anxiety in military veterans with chronic obstructive pulmonary diseae. Depress Anxiety 2006; 23: 42-49.

8 Hill K, Geist R, Goldstein RS, et al. Anxiety and depression in end-stage COPD. Eur Respir J 2008; 31: 667-677.

9 Bhandari NJ, Jain T, Marolda C, et al. Comprehensive pulmonary rehabilitation results in clinically meaningful improvements in anxiety and depression in patients with chronic obstructive pulmonary disease. $J$ Cardiopulm Rehabil Prev 2013; 33: 123-127.

10 Yohannes AM, Dryden S, Hanania NA. The responsiveness of the Anxiety Inventory for Respiratory Disease scale following pulmonary rehabilitation. Chest 2016; 150: 188-195.

11 Cully JA, Stanley MA, Petersen NJ, et al. Delivery of brief cognitive behavioral therapy for medically ill patients in primary care: a pragmatic randomized clinical trial. J Gen Intern Med 2017; 32: 1014-1024.

12 Usmani ZA, Carson KV, Heslop K, et al. Psychological therapies for the treatment of anxiety disorders in chronic obstructive pulmonary disease. Cochrane Database Syst Rev 2017; 3: CDO10673.

13 Beck JS. Cognitive behavioral therapy: basics and beyond. 2nd Edn. New York, The Guilford Press, 2011.

14 Hynninen MJ, Bjerke N, Pallesen S, et al. A randomized controlled trial of cognitive behavioral therapy for anxiety and depression in COPD. Respir Med 2010; 104: 986-994.

15 Kunik ME, Veazey C, Cully JA, et al. COPD education and cognitive behavioral therapy group treatment for clinically significant symptoms of depression and anxiety in COPD patients: a randomized controlled trial. Psychol Med 2008; 38: 385-396.

16 Hazell CM, Greenwood K, Fielding-Smith S, et al. Understanding the barriers to accessing symptom-specific cognitive behavioral therapy (CBT) for distressing voices: reflecting on and extending the lessons learnt from the CBT psychosis literature. Front Psychol 2018; 9: 727.

17 Yohannes AM. General practitioners views and experiences in managing depression in patients with chronic obstructive pulmonary disease. Expert Rev Resp Med 2012; 6: 589-595. 
18 Jiang X, He G. Effects of an uncertainty management intervention on uncertainty, anxiety, depression and quality of life of chronic obstructive pulmonary disease outpatients. Res Nurs Health 2012; 35: 409-418.

19 Smith SMS, Sonego S, Ketcheson L, et al. A review of the effectiveness of psychological interventions used for anxiety and depression in chronic obstructive pulmonary disease. BMJ Open Res 2014; 1: e000042.

20 Heslop-Marshall K, Baker C, Carrick-Sen D, et al. Randomised controlled trial of cognitive behavioural therapy in COPD. ERJ Open Research 2018; 4: 00094-2018.

21 Lewis C, Pearce J, Bisson JI. Efficacy, cost-effectiveness and acceptability of self-help interventions for anxiety disorders: systematic review. Br J Psychiatry 2012; 200: 15-21.

22 Cuijpers P, Donker T, van Straten A, et al. Is guided self-help as effective as face-to-face psychotherapy for depression and anxiety disorders. A systematic review and meta-analysis of comparative studies. Psychol Med 2010; 40: 1943-1957.

23 Hundt NE, Renn BN, Sansgiry S, et al. Predictors of response to brief CBT in patients with cardiopulmonary conditions. Health Psychol 2018; 37: 866-873.

24 Maurer J, Rebbapragada V, Borson S, et al. Anxiety and depression in COPD: current understanding, unanswered questions, and research needs. Chest 2008; 134: 43S-56S. 